\title{
PUERICULTURA NA ATENÇÃO PRIMÁRIA À SAÚDE: ATUAÇÃO DO ENFERMEIRO
}

Viviane Cazetta de Lima Vieiraㄹ, Claudiane Amaro Fernandes ${ }^{2}$, Marcela de Oliveira Demitto ${ }^{2}$, Luciana Olga Bercini Maria José Scochi ${ }^{4}$, Sonia Silva Marcon ${ }^{5}$

RESUMO: Objetivou-se com este estudo conhecer aspectos relacionados à atuação do enfermeiro na puericultura. Tratase de uma pesquisa descritiva exploratória de natureza qualitativa. Os informantes foram todos os enfermeiros atuantes nas Unidades Básicas de Saúde de Mandaguari-Paraná e os dados foram coletados em 2010 por meio de entrevista semiestruturada e após, submetidos à análise de conteúdo. Sobre o tema emergiram duas categorias: Puericultura: atividades envolvidas, a qual mostra ser essa uma oportunidade para orientar sobre alimentação, imunização e higiene e acompanhar o crescimento da criança e Organização da puericultura no município: entre a prática e a pretensão, a qual aponta dificuldades de operacionalização, como falta de capacitação, ausência de protocolo e falhas no processo de referência e contra-referência. As discussões ensejaram reflexões a respeito da prática da enfermagem em puericultura, o que poderá contribuir para a elaboração de novas estratégias no acompanhamento de saúde da criança no município.

PALAVRAS-CHAVE: Saúde da criança; Atenção primária à saúde; Enfermagem.

\section{CHILDCARE IN PRIMARY HEALTHCARE: THE NURSE'S ROLE}

ABSTRACT: This study aimed to shed light on aspects related to the role of the nurse in childcare. It is descriptive, exploratory research, of a qualitative nature. Data was provided by all the nurses working in the basic Health Units in Mandaguari-Paraná, was collected in 2010 through semi-structured interviews, and was later submitted to content analysis. Two categories emerged about the theme: childcare and activities it involves, which shows that this is an opportunity to provide guidance on nutrition, immunization and hygiene, and on accompanying the growth of the child, and organization of childcare in the town: between plan and practice, which highlights difficulties of operationalization, such as lack of enablement, absence of protocols, and flaws in the process of reference and counter-reference. The discussions provided an opportunity for reflections about nursing practice in childcare, which can contribute to the development of new strategies for monitoring children's health in the town. KEYWORDS: Children's health; Primary Healthcare; Nursing.

\section{PUERICULTURA EN LA ATENCIÓN PRIMARIA A LA SALUD: ACTUACIÓN DEL ENFERMERO}

RESUMEN: El objetivo de este estudio fue conocer aspectos relacionados a la actuación del enfermero en la puericultura. Es una investigación descriptiva exploratoria de naturaleza cualitativa. Los informantes fueron todos enfermeros actuantes en las Unidades Básicas de Salud de Mandaguari - Paraná y los datos fueron recogidos en 2010 por medio de entrevista semiestructurada y sometidos al análisis de contenido. Acerca del tema, surgieron dos categorías: Puericultura: actividades involucradas, la cual muestra ser esa una oportunidad de orientar sobre alimentación, imunización y higiene así como acompañar el crecimiento del niño y Organización de la puericultura en municipio: entre la práctica y la pretensión, la cual apunta dificultades de operacionalización, como falta de capacitación, ausencia de protocolo y problemas en el proceso de referencia y contrareferencia. Las discusiones provocaron reflexiones acerca de la práctica de la enfermería en puericultura, lo que podrá contribuir para la elaboración de nuevas estrategias en el acompañamiento de salud del niño en el municipio. PALABRAS-CLAVE: Salud del niño; Atención primaria a la salud; Enfermería.

${ }^{1}$ Enfermeira. Mestranda em Enfermagem pela Universidade Estadual de Maringá - UEM. Professora da Faculdade de Filosofia Ciências e Letras de Mandaguari.

${ }^{2}$ Enfermeira. Mestranda em Enfermagem pela UEM.

${ }^{3}$ Enfermeira. Doutora em Ecologia de Ambientes Aquáticos Continentais. Professora do Curso de Graduação e do Programa de PósGraduação em Enfermagem da UEM.

${ }^{4}$ Enfermeira. Doutora em Saúde Pública. Professora do Curso de Graduação e do Programa de Pós-Graduação em Enfermagem da UEM.

${ }^{5}$ Enfermeira. Doutora em Filosofia da Enfermagem. Coordenadora do Mestrado em Enfermagem da UEM. Coordenadora do NEPAAF - Núcleo de Estudos, Pesquisa, Assistência e Apoio à família.

Autor correspondente:

Recebido: 01/11/2011

Viviane Cazetta de Lima Vieira

Aprovado: 15/02/2012

Universidade Estadual de Maringá

Rua Romário Martins, 80 - CEP - Mandaguari-PR-Brasil

E-mail: vivianecazettadelimavieira@gmail.com 


\section{INTRODUÇÃO}

A infância é um período em que se desenvolve grande parte das potencialidades humanas. No entanto, os distúrbios que incidem nessa época são responsáveis por graves consequências para os indivíduos e comunidades. Assim, ações vêm sendo exploradas a fim de garantir e manter a qualidade de vida dessa população ${ }^{(1)}$. Para que a criança cresça de maneira saudável e esteja preparada para enfrentar as transformações que ocorrem em seu organismo, é necessário que ela receba cuidados específicos, capazes de promover seu bem estar físico e prevenir problemas que possam interferir em seu desenvolvimento neuropsicomotor ${ }^{(2)}$.

$\mathrm{Na}$ área da Atenção Primária à Saúde, responsável pela prevenção, promoção e recuperação da saúde dos indivíduos em todas as fases da vida, a Estratégia Saúde da Família (ESF), desde a sua criação no ano de 1993, vem se consolidando como um dos eixos estruturantes do Sistema Único de Saúde (SUS), por meio de movimento de expressiva expansão de cobertura populacional, aprimorando em muito o acesso da população às ações de saúde ${ }^{(1)}$.

Nesta Estratégia, um dos instrumentos utilizados para o acompanhamento da saúde das crianças é o Programa de Puericultura, que tem como propósito acompanhar o crescimento e desenvolvimento, observar a cobertura vacinal, estimular a prática do aleitamento materno, orientar a introdução da alimentação complementar e prevenir as doenças que mais freqüentemente acometem as crianças no primeiro ano de vida, como a diarréia e as infecções respiratórias ${ }^{(3)}$.

O Ministério da Saúde, a fim de garantir a qualidade da assistência prestada à criança, propõe um calendário mínimo de consultas de puericultura, assim distribuídas: uma consulta até 15 dias de vida, consultas com um mês, dois, quatro, seis, doze e dezoito meses, totalizando assim, sete consultas no primeiro ano e meio de vida( ${ }^{(2)}$.

A puericultura é desenvolvida tanto pelo médico quanto pelo enfermeiro. As atribuições do enfermeiro neste programa são: realizar o exame físico na criança, identificando riscos em seu crescimento e desenvolvimento; agendar a primeira consulta com o pediatra e demais quando forem identificados riscos de agravos à saúde; fornecer a relação dos nascidos vivos para os Agentes Comunitários de Saúde (ACS) e solicitar a busca ativa para identificação dos faltosos do programa; preencher o gráfico de peso e estatura nos cartões da criança; verificar e administrar as vacinas conforme o calendário básico de vacinação; incentivar o Aleitamento Materno Exclusivo (AME) até o seis meses; orientar a alimentação complementar após os seis meses; orientar sobre prevenção de acidentes de acordo com a faixa etária; avaliar o desenvolvimento neuropsicomotor; identificar dúvidas e dificuldades da mãe e de outros membros da família que participam das consultas procurando esclarecê-las ${ }^{(2)}$.

Dentro desta perspectiva, o enfermeiro, por meio de revisões periódicas, desempenha seu trabalho com ações não apenas clínicas, mas com uma concepção epidemiológica e social, relacionando-as intimamente com o complexo saúde-indivíduo-família-comunidade.

Sabendo desta complexidade, o presente estudo teve como objetivo conhecer aspectos relacionados à atuação do enfermeiro em puericultura na atenção primária à saúde do município de Mandaguari-PR. Pretende-se com tal conhecimento contribuir com a elaboração de novas estratégias de atuação do enfermeiro no acompanhamento de saúde da criança no município em questão.

\section{MÉTODO}

Trata-se de um estudo qualitativo, desenvolvido no município de Mandaguari, situado na região noroeste do estado do Paraná, localizado a $400 \mathrm{~km}$ de sua capital - Curitiba, com população de 32.669 habitantes em 2010. O município conta com seis Unidades Básicas de Saúde (UBS) e todas elas utilizam a ESF.

Foram inclusos na pesquisa todos os 8 enfermeiros que trabalhavam nas seis UBS do município, no período do estudo - outubro de 2010, por meio de entrevistas semiestruturadas. O instrumento utilizado na coleta de dados foi um roteiro contendo questões fechadas sobre o perfil sociodemográfico do enfermeiro, e questões abertas sobre a operacionalização da puericultura na UBS e sobre o conhecimento do profissional sobre a mesma. As entrevistas foram previamente agendadas e ocorreram na própria UBS de atuação do enfermeiro, em sala reservada, e tiveram duração média de 20 minutos. A fim de garantir melhor interação entre entrevistador e os entrevistados e proporcionar maior agilidade, as entrevistas foram gravadas, sendo posteriormente transcritas na íntegra pelos pesquisadores para análise dos dados. Após esta etapa, os dados passaram por processo analítico e descritivo a partir do referencial de análise de conteúdo de $\operatorname{Bardin}^{(4)}$.

O desenvolvimento do estudo ocorreu em conformidade com o preconizado pela Resolução 196/96 do Conselho Nacional de Saúde. O projeto de pesquisa foi 
aprovado pelo Comitê Permanente de Ética em Pesquisa com Seres Humanos da Universidade Estadual de Maringá (Parecer n. 591/2010).

\section{RESULTADOS}

A maioria dos enfermeiros em estudo (seis) é do sexo feminino, jovens, com idade entre 23 e 30 anos. Em relação ao tempo de formação profissional um enfermeiro possui nove meses de formado e os demais (sete) entre um e quatro anos de formação. Acerca do tempo de serviço no trabalho atual as respostas variaram de três meses a três anos, destacando que para a maioria (seis) este é o primeiro emprego.

Em relação ao conhecimento dos aspectos relacionados à atuação desses profissionais no Programa de Puericultura foram elencadas duas categorias: Compreensão do enfermeiro sobre puericultura e Organização da puericultura no município: entre a prática e a pretensão.

\section{Compreensão do enfermeiro sobre puericultura}

No discurso dos enfermeiros sobre a compreensão do que é o Programa de Puericultura percebe-se que em todas as falas o conceito de puericultura esteve atrelado à prática de orientar, como os recortes abaixo.

Puericultura eu entendo que não é somente pesar e medir. Nós fazemos orientações sobre alimentação, vacinas, cuidados. (E1)

Eu vejo as medidas, faço a inspeção e depois anoto no prontuário. Além disto, eu faço orientações. (E1)

A gente verifica o peso, perimetro cefálico e abdominal, altura e anota no cartão de vacina. Explicamos para a mãe que a criança está crescendo, se desenvolvendo através de orientações. (E2)

$\mathrm{O}$ ato de orientar, segundo os entrevistados, pode ser compreendido também como um momento para realizar a educação em saúde eneste sentido a família está diretamente relacionada devendo ser ouvida e acolhida, como a seguir:

É um momento em que estão presentes mãe e filho, ou o pai também. É um atendimento individual para identificar se a criança está bem. (E1)

A puericultura esteve relacionada também a um momento dedicado a avaliação do estado nutricional infantil. Neste sentido, as orientações sobre as questões relativas à amamentação e alimentação foram destacadas pelos enfermeiros entrevistados nos depoimentos:

[...] é a pesagem da criança, o estado nutricional que ela se encontra. Às vezes a gente vê somente a questão da pesagem e não é, é o estado nutricional também. (E5)

As consultas são importantes para incentivar e atuar principalmente no aleitamento materno e orientar alimentação saudável. (E8)

As falas revelaram que a verificação da carteira de vacinação da criança, assim como a realização de orientações sobre o assunto, são ações de grande importância atribuída pelo profissional enfermeiro:

É muito importante saber se a criança está recebendo as vacinas. A gente faz a orientação dependendo do questionamento da mãe, sempre pegando no pé com relação às vacinas. (E6)

Questões referentes à higiene da criança foram pontuadas como um tema oportuno a ser abordado no acompanhamento adequado da puericultura:

Conforme vai indo, eu oriento sobre a higiene intima da criança. (E3)

A gente vai orientando sobre a higiene, como lavar bem as mãos durante a alimentação. (E8)

Cabe destacar também que alguns aspectos importantes da saúde da criança não foram citados pelos profissionais, tais como prevenção de acidentes na infância, violência domiciliar, cuidados com o coto umbilical, icterícia neonatal, teste do pezinho, doenças prevalentes da infância, entre outros. Fato este preocupante, já que a puericultura consiste em momento adequado para realizar a educação em saúde e assim promover a saúde infantil.

\section{Organização da puericultura no município: entre a prática e a pretensão}

No município em estudo não há um protocolo referente à consulta de puericultura, como mostra as falas:

[...] nós fazemos a puericultura no puerpério ou quando as crianças vêm fazer vacina ou pesar para o leite 
[referindo-se ao Programa do Leite]. (E7)

Eu faço assim, de zero a dois anos é mensal. Depois dos dois anos, não faz mais. (E1)

Se a criança é baixo peso, a cada 15 dias; se é normal, a cada um mês até o sexto mês. A partir do sexto mês, ai é a cada dois meses. (E5)

No que tange à captação da criança para a consulta de puericultura, ou seja, a estratégia para incluir a criança no Programa, o ACS foi citado como o principal responsável por tal ação, como exemplos abaixo:

Os ACS fazem uma pesquisa das crianças, a captação é mais por parte deles. (E1)

\section{É feito a busca ativa pelos ACS. (E3)}

Os enfermeiros assinalaram que, em algumas situações, necessitam realizar o encaminhamento das crianças acompanhadas à consulta médica, sendo alguns destes motivos mencionados nas seguintes falas:

Bom, depende pelo exame físico. Se tem alguma coisa anormal, se o peso está muito abaixo, ai a gente procura passar pelo pediatra. (E1)

Se está com febre, abaixo do peso, sobrepeso, às vezes eu até já marco a consulta. (E6)

Geralmente, dermatite, hérnia [...]. (E7).

Porém, ao encaminhar a criança para o pediatra, foi relatado por todos os enfermeiros a não ocorrência de contra-referência.

Não, ele [o pediatra] nunca fez a contra-referência. (E4)

O pediatra atende bastante, então não tem nada detalhado no prontuário, não. Normalmente é com a mãe que a gente acaba conseguindo algum dado. (E5)

Segundo a fala dos entrevistados, a capacitação profissional dentro da ESF, mesmo constituindo ferramenta fundamental, foi revelado como insuficiente, sendo remetidas somente às de aleitamento materno, como relato a seguir:

Capacitação [...] não, nada. De puericultura? Ah, eu tive de aleitamento materno. (E7)
Não, só de aleitamento materno. (E6)

De maneira geral, a consulta de puericultura foi considerada pelos enfermeiros como muito importante na atenção à saúde da criança. Os sujeitos do estudo destacaram que o valor das consultas está ligado à oportunidade de realizar a avaliação infantil, como demonstrado nas falas:

Eu acho muito importante porque assim, a gente pode estar observando todo o crescimento e o desenvolvimento, e a gente pode ver algum tipo de doença, detectando algum problema. (E3)

A puericultura é um programa de assistência individualizada à criança, que consiste no acompanhamento do crescimento e o desenvolvimento nos primeiros anos de vida. (E8)

\section{DISCUSSÃO}

O estudo revelou que a concepção dos enfermeiros no município sobre o programa de puericultura é uma questão ampla e que não se restringe a pesagem e verificação de medidas antropométricas, mas possui ênfase na orientação acerca de questões relacionadas à saúde da criança.

As orientações estão pautadas em ações que envolvem a educação em saúde. Este é um importante instrumento de trabalho, principalmente quando pautada na problematização da realidade, na busca da conscientização e na construção de indivíduos críticos, oportunizando a troca de saberes, no qual o conhecimento profissional e o senso comum se unem em nome do bom senso ${ }^{(5)}$.

Nesse sentido, a ESF tem buscado reorganizar a atenção básica, propondo que a educação em saúde seja centrada na família, a qual é vista e entendida a partir do seu ambiente físico e social, enfatizando uma compreensão ampliada do processo saúde-doença e da necessidade de intervenções que vão para além das práticas curativas.

Assim, é fundamental considerar como tarefa indispensável do enfermeiro a transmissão de conhecimentos por meio das orientações nas consultas de puericultura, além de buscar ampliar a autonomia da mãe e reforçar sua condição de sujeito social, a fim de torná-la capaz de prestar o melhor cuidado ao seu filho.

Este suporte dos serviços de saúde dirigido às mães pode contribuir entre outras ações para melhorar a condução no processo alimentar de seus filhos. Esta 
abordagem é efetivada a partir da consulta de enfermagem em puericultura, sendo o enfermeiro participante ativo desse processo ${ }^{(6)}$.

Estudo realizado com 112 crianças residentes na área de abrangência de uma UBS no Rio Grande do Sul, sobre o impacto do programa de puericultura e a prevalência de aleitamento materno, destacou que as taxas de AME no sexto mês foram superiores às taxas nacionais ${ }^{(7)}$. Vale destacar que na UBS estudada tem-se como rotina do Programa de Puericultura a realização de visitas domiciliares às mães no pós-parto imediato para apoio quanto à dificuldade na amamentação, e consultas mensais às crianças no primeiro ano de vida, práticas essas que aumentam o contato da mãe com os profissionais de saúde.

Neste sentido, o vínculo entre o usuário e o serviço de saúde se torna eficaz nas ações de saúde e auxilia na participação do usuário na continuidade da procura pelos serviços de saúde. Vale enfatizar também que o vínculo ajuda na formação da autonomia tanto dos usuários como dos próprios profissionais ${ }^{(8)}$.

O vínculo também contribui de forma significativa para o alcance de metas de programas como a vacinação, trazendo importante impacto sobre as doenças imunopreveníveis, resultando em mudanças no padrão de adoecimento e morte especialmente da população infantil brasileira ${ }^{(9)}$.

Embora haja consenso entre os enfermeiros entrevistados sobre a importância da vacinação, dados preliminares da cobertura vacinal em menores de um ano em Mandaguari- PR mostram valores aquém daqueles encontrados na $15^{\mathrm{a}}$ Regional de Saúde, regional à qual o município em estudo pertence. A cobertura vacinal na $15^{\text {a }}$ Regional apresenta a seguinte cobertura vacinal: BCG $(112,02 \%)$, hepatite B $(104,85 \%)$, pólio $(106,10 \%)$ e tetra $(106,17 \%)$ enquanto os dados de Mandaguari são: BCG $(92,09 \%)$, hepatite B $(87,44 \%)$, pólio $(87,44 \%)$ e tetra $(86,5 \%)^{(10)}$. Entretanto, sabe-se que a cobertura vacinal não está atrelada somente a um evento. Vários fatores têm sido associados a disparidades nas coberturas vacinais entre populações e regiões como: características individuais e familiares, características do contexto onde as pessoas vivem e características dos serviços que desenvolvem as atividades de vacinação ${ }^{(11)}$.

Estudo realizado com 62 enfermeiros atuantes nas UBS de município nordestino mostrou que as principais dificuldades enfrentadas por esses profissionais, para alcançar a meta de $100 \%$ de vacinação infantil proposta pelo Programa Nacional de Imunização, foram a resistência dos pais à vacinação, o que pode estar associado às crenças, cultura dos mais velhos e, até mesmo, ao medo da reação da vacina; ainda, falta de ACS considerando que em áreas descobertas por eles não é possível saber o número real de crianças menores de um ano, além da falta de material ${ }^{(12)}$.

Assim, as ações de investigação da situação vacinal infantil e de orientação à comunidade sobre o assunto, realizadas durante as consultas de puericultura pelos profissionais da presente pesquisa, podem ser considerados como um dos passos para se atingir índices mais elevados de cobertura vacinal no município.

Além da vacinação, outras medidas podem ser adotadas no combate a patologias prevalentes na infância, como é o caso da higiene do recém nascido. Dessa forma, as orientações sobre os cuidados com a higiene, levando-se em consideração as condições de moradia, educação, cultura e renda da família, são fundamentais na prevenção de doenças ${ }^{(2)}$ e devem ser abordadas nas consultas de puericultura.

Nota-se diante do exposto que a compreensão dos enfermeiros sobre puericultura é fruto da vivência cotidiana em seu ambiente de trabalho, o que lhes permite considerar tal consulta sempre atrelada às práticas educativas, revelando-se como momento oportuno para orientar a mãe e/ou a família sobre questões relacionadas à saúde da criança.

Porém, percebe-se nos discursos o não-aprofundamento das orientações realizadas durante a puericultura. Os enfermeiros não detalharam quais orientações costumam fornecer dentro de cada grupo citado por eles, como amamentação, vacinação, higiene, tornando as falas um tanto superficiais, o que pode sugerir timidez, pelo fato da entrevista estar sendo gravada, limitação de tempo ou ainda falta de preparo técnico-científico.

Assim, como o não aprofundamento das orientações, notou-se que alguns aspectos não foram evidenciados nas falas como foi o caso da identificação de uma criança de risco a qual é ponto chave no atendimento à criança. Este grupo deve ser priorizado para o desenvolvimento das ações de vigilância à saúde segundo proposta de acompanhamento do crescimento e desenvolvimento da criança menor de cinco anos e segundo protocolos específicos (atenção ao desnutrido, criança com asma, entre outros) ${ }^{(13)}$.

Esta assistência deve estar desenhada de forma a compor a rede integral de saúde da criança, de forma regionalizada, permitindo o acesso e promovendo sua qualidade de vida. A responsabilidade das equipes de saúde da família se mantém sobre essa população de maior risco, provendo a continuidade do cuidado e desenvolvendo ações de vigilância à saúde. 
Situações como estas descritas podem estar vinculadas à falta de um protocolo referente às consultas de puericultura, ou seja, um documento que possa nortear as ações desses profissionais de saúde, o qual não existe no município. A falta deste material dificulta a padronização de condutas entre as UBS como, por exemplo, o intervalo entre as consultas no calendário da criança sadia e da criança de risco, e a forma de captação da criança para a consulta de puericultura.

A captação das crianças para o acompanhamento de seu crescimento de desenvolvimento é realizada, na maioria das vezes, pelo ACS. Este, principal elo entre a Unidade de Saúde da Família (USF) e a comunidade, tem a possibilidade de abordagem da criança nos espaços de sua vida cotidiana (domicílio e instituições de educação infantil) ampliando dessa forma, a capacidade de atuação na prevenção de doenças, na promoção da saúde e identificação de necessidades especiais em termo oportuno ${ }^{(13)}$. Contudo, o enfermeiro como líder da equipe também precisa estar envolvido em todo o processo de captação dessas crianças, mantendo a equipe atualizada sobre o número de gestantes e de nascidos vivos em cada área de abrangência.

Outro fator revelado nas falas é a falta de comunicação entre os profissionais de saúde no cuidado à criança, o que prejudica a integralidade do cuidado prejudicando o atendimento prestado à criança $\mathrm{e}$ sua família. A atuação em equipe é definida como a articulação de diversos saberes e intervenções dos profissionais da unidade de saúde, efetivando o trabalho solidário e compartilhado e produzindo resposta qualificada às necessidades em saúde da criança ${ }^{(13)}$. Quando este princípio é rompido, a assistência prestada pode ficar comprometida.

$\mathrm{Na}$ ESF, base da atenção primária, muitos problemas de saúde podem ser solucionados ou amenizados. Porém, para que isto aconteça, faz-se necessário a capacitação dos profissionais, o que foi revelado como insuficiente pelos enfermeiros da presente pesquisa.

A capacitação teórico-prática e a supervisão da educação continuada das equipes de saúde da família e de atenção básica são fundamentais para a plena inserção de todos os profissionais no cuidado com a criança. A abordagem neste sentido deve ter como eixo a visão global da criança, enfocando a identificação daquela com maior vulnerabilidade e risco, de forma a orientar a priorização do atendimento para a criança em algum sinal de gravidade, o aconselhamento da mãe-família e a responsabilização pela continuidade da assistência com acompanhamento até a solução dos problemas apresentados ${ }^{(13)}$.
De maneira geral a consulta de puericultura foi considerada pelos enfermeiros como muito importante na atenção à saúde da criança. Os sujeitos do estudo destacaram que o valor das consultas está ligado à oportunidade de realizar a avaliação infantil. $\mathrm{O}$ acompanhamento do crescimento e desenvolvimento faz parte da avaliação integral à saúde da criança e envolve o registro no Cartão da Criança, de avaliação de peso, altura, desenvolvimento, vacinação e intercorrências, o estado nutricional, bem como orientações à mãe/ família/cuidador sobre os cuidados com a criança em todo atendimento ${ }^{(13)}$.

O crescimento representa um dos fatores mais importantes da saúde da criança, sendo considerado como uma forma importante de conhecer e vigiar o estado geral da saúde de uma criança e o desenvolvimento sócio econômico e de saúde da comunidade onde ela vive ${ }^{(14)}$.

Parte desta avaliação é realizada por meio da verificação das medidas antropométricas. O enfermeiro deve ter consciência da importância da mensuração das crianças na puericultura, uma vez que estes refletem o estado de saúde da população infantil sob sua responsabilidade e permite evidenciar de maneira precoce os transtornos que afetam a saúde, a nutrição e o desenvolvimento da criança ${ }^{(14)}$.

É imprescindível a detecção de déficits de peso e/ou estatura e também de casos de obesidade, a fim de evitar comprometimentos futuros à saúde da criança. Nesta perspectiva, a consulta de puericultura mostra-se, mais uma vez, como um instrumento de triagem na detecção precoce de riscos e agravos ao desenvolvimento infantil, além de ser o cenário no qual o profissional enfermeiro pode orientar pais e familiares na busca de promover um ambiente seguro e facilitador para que a criança se desenvolva de maneira saudável ${ }^{(15)}$.

\section{CONSIDERAÇÕES FINAIS}

A puericultura, sem dúvida, consiste em uma ferramenta oportuna para a realização de educação em saúde e para o acompanhamento integral do crescimento e desenvolvimento infantil. Embora, o acompanhamento da puericultura aconteça na atenção primária de saúde do município estudado, os enfermeiros revelaram algumas dificuldades de operacionalização, como falta de preparo técnico-científico, evidenciada nas falas pela falta de capacitação, ausência de um protocolo que embase suas ações e falhas no processo de comunicação para referência e contra-referência 
entre os profissionais da própria unidade, dificultando a continuidade da assistência à criança e sua família.

Faz-se necessário modificar esta realidade com o objetivo de difundir a importância do seguimento periódico e contínuo dos programas de puericultura, estimulando os pais a procurarem, com assiduidade, as unidades de saúde, e diante das intercorrências fazê-la o mais precoce possível.

Por fim, com uma política que inclua a qualificação dos profissionais de saúde que atuam nestas UBS, ações padronizadas nos município, por meio da elaboração de protocolos e criação de um sistema de referência e contra-referência, aliado ao esforço conjunto de gestores e profissionais e uma visão integrada da saúde acredita-se que os problemas identificados possam ser superados. Dessa forma é possível proporcionar a todas as crianças chances de atingir todo seu potencial de crescimento e desenvolvimento, e consequentemente uma melhor qualidade de vida futura.

\section{REFERÊNCIAS}

1. Brasil. Ministério da Saúde. Secretaria de Atenção à Saúde. Departamento de Atenção Básica. Saúde da criança: nutrição infantil: aleitamento materno e alimentação complementar - Brasília: Editora do Ministério da Saúde; 2009.

2. Londrina. Prefeitura do Município. Autarquia Municipal de Saúde. Saúde da criança: protocolo. 1. ed.- Londrina, PR; 2006.

3. Leite GB, Bercini LO. Caracterização das crianças atendidas na puericultura do programa saúde da família do município de Campo Mourão, Paraná, em 2003. Cienc. cuid. saude. 2005;4(3):224-30.

4. Bardin L. Análise de conteúdo. $4^{\mathrm{a}}$ ed. Lisboa: Edições; 2009.

5. Acioli S. A prática educativa como expressão do cuidado em saúde pública. Rev. bras. enferm. 2008;61(1):117-21.

6. Santos CS, Lima LS, Javorski M. Fatores que interferem na transição alimentar de crianças entre cinco e oito meses: investigação em Serviço de Puericultura do Recife, Brasil. Rev. bras saude mater. infant. 2007;7(4):373-80.

7. Faleiros JJ, Kalil G, Casarin DP, Laque PA, Santos IS. Avaliação do impacto de um programa de puericultura na promoção da amamentação exclusiva. Cad. saude publica. 2005;21(2):482-9.
8. Rangel RF, Fugali MM, Backes DS, Gehlen H, Souza HT. Avanços e perspectivas da atuação do enfermeiro em estratégia saúde da família. Cogitare enferm. 2001;16(3):498-504.

9. Brasil. Ministério da Saúde. Secretaria de Vigilância em saúde. Síntese da situação epidemiológica e operacional da vigilância das doenças de transmissão respiratória e imunopreveníveis no Brasil. Brasília; 2006.

10. Secretaria do Estado de Saúde do Paraná. SESA/SVS/ DEVE [Internet]. [acesso em 07 dez 2010]. Disponível: www.sesa.pr.gov.br.

11. Luhm KR. Fatores associados à situação vacinal em Curitiba [tese]. São Paulo: Faculdade de Saúde Pública da Universidade de São Paulo; 2008.

12. França ISX, Alves FP, Brito VRS. Cobertura vacinal e mortalidade infantil em Campina Grande, PB, Brasil. Rev. bras. enferm. 2009;62(2):258-64.

13. Ministério da Saúde (BR). Agenda de compromissos para a saúde integral da criança e redução da mortalidade infantil. Brasília (DF): Ministério da Saúde; 2005.

14. Fujimore E, Ohara CVS. Enfermagem e a saúde da criança na atenção básica. São Paulo: Manole. 2009.

15. Santos MEA, Quintão NT, Almeida RX. Avaliação dos marcos do desenvolvimento infantil segundo a estratégia da atenção integrada às doenças prevalentes na infância. Esc. Anna Nery. 2010;14(3):591-8. 